\title{
Osteopontin and Disease Activity in Patients with Recent-onset Systemic Lupus Erythematosus: Results from the SLICC Inception Cohort
}

Lina Wirestam (1D, Helena Enocsson (1), Thomas Skogh, Leonid Padyukov, Andreas Jönsen, Murray B. Urowitz, Dafna D. Gladman, Juanita Romero-Diaz, Sang-Cheol Bae, Paul R. Fortin, Jorge Sanchez-Guerrero, Ann E. Clarke, Sasha Bernatsky, Caroline Gordon, John G. Hanly, Daniel Wallace, David A. Isenberg, Anisur Rahman, Joan Merrill, Ellen Ginzler, Graciela S. Alarcón, W. Winn Chatham, Michelle Petri, Munther Khamashta, Cynthia Aranow, Meggan Mackay, Mary Anne Dooley, Susan Manzi, Rosalind Ramsey-Goldman, Ola Nived, Kristjan Steinsson, Asad Zoma, Guillermo Ruiz-Irastorza, Sam Lim, Ken Kalunian, Murat Inanc, Ronald van Vollenhoven, Manuel Ramos-Casals, Diane L. Kamen, Søren Jacobsen, Christine Peschken, Anca Askanase, Thomas Stoll, Ian N. Bruce, Jonas Wetterö ${ }^{\mathbb{D}}$, and Christopher Sjöwall ${ }^{\mathbb{D}}$

ABSTRACT. Objective. In cross-sectional studies, elevated osteopontin (OPN) levels have been proposed to reflect, and/or precede, progressive organ damage and disease severity in systemic lupus erythematosus (SLE). We aimed, in a cohort of patients with recent-onset SLE, to determine whether raised serum OPN levels precede damage and/or are associated with disease activity or certain disease phenotypes. Methods. We included 344 patients from the Systemic Lupus International Collaborating Clinics (SLICC) Inception Cohort who had 5 years of followup data available. All patients fulfilled the 1997 American College of Rheumatology (ACR) criteria. Baseline sera from patients and from age- and sex-matched population-based controls were analyzed for OPN using ELISA. Disease activity and damage were assessed at each annual followup visit using the SLE Disease Activity Index 2000 (SLEDAI-2K) and the SLICC/ACR damage index (SDI), respectively.

Results. Compared to controls, baseline OPN was raised 4-fold in SLE cases $(\mathrm{p}<0.0001)$. After relevant adjustments in a binary logistic regression model, OPN levels failed to significantly predict global damage accrual defined as SDI $\geq 1$ at 5 years. However, baseline OPN correlated with SLEDAI-2K at enrollment into the cohort $(\mathrm{r}=0.27, \mathrm{p}<0.0001)$, and patients with high disease activity (SLEDAI- $2 K \geq 5)$ had raised serum OPN $(\mathrm{p}<0.0001)$. In addition, higher OPN levels were found in patients with persistent disease activity $(\mathrm{p}=0.0006)$, in cases with renal involvement $(\mathrm{p}<0.0001)$ and impaired estimated glomerular filtration rate $(\mathrm{p}=0.01)$.

Conclusion. The performance of OPN to predict development of organ damage was not impressive. However, OPN associated significantly with lupus nephritis and with raised disease activity at enrollment, as well as over time. (First Release January 15 2019; J Rheumatol 2019;46:492-500; doi:10.3899/jrheum.180713)

Key Indexing Terms:

SYSTEMIC LUPUS ERYTHEMATOSUS BIOMARKERS DISEASE ACTIVITY

ORGAN DAMAGE

OSTEOPONTIN

PROGNOSIS

From the Rheumatology/Division of Neuro and Inflammation Sciences, Department of Clinical and Experimental Medicine, Linköping University, Linköping; Department of Medicine, Unit of Rheumatology, Karolinska Institutet and Karolinska University Hospital, Stockholm; Department of Clinical Sciences Lund, Section of Rheumatology, Lund University, Lund; Unit for Clinical Therapy Research (ClinTRID), Karolinska University, Stockholm, Sweden; Centre for Prognosis Studies in the Rheumatic Diseases, Toronto Western Hospital and University of Toronto, Toronto, Ontario; Division of Rheumatology, Centre Hospitalier Universitaire (CHU) de Québec - Université Laval, Quebec City, Quebec; Division of Rheumatology, Cumming School of Medicine - University of Calgary, Calgary, Alberta; Division of Rheumatology, Department of Medicine, McGill University Health Centre, Montreal, Quebec; Division of
Rheumatology, Department of Medicine and Department of Pathology, Queen Elizabeth II Health Sciences Centre and Dalhousie University, Halifax, Nova Scotia; Department of Medicine and Community Health Sciences, University of Manitoba, Winnipeg, Manitoba, Canada; Instituto Nacional de Ciencias Médicas y Nutrición, Mexico City, Mexico; Department of Rheumatology, Hanyang University Hospital for Rheumatic Diseases, Seoul, Korea; Rheumatology Research Group, School of Immunity and Infection, College of Medical and Dental Sciences, University of Birmingham, Birmingham; Centre for Rheumatology Research, University College, London; Lupus Research Unit, The Rayne Institute, St. Thomas' Hospital, King's College London School of Medicine, London; Lanarkshire Centre for Rheumatology, Hairmyres Hospital, East Kilbride, Scotland; Arthritis Research UK Centre for 
Epidemiology, Centre for Musculoskeletal Research, Faculty of Biology, Medicine and Health, The University of Manchester and UK National Institute for Health Research (NIHR) Manchester Biomedical Research Centre, Manchester University Foundation Trust, Manchester, UK; Cedars-Sinai/David Geffen School of Medicine at University of California at Los Angeles, Los Angeles, California; Department of Clinical Pharmacology, Oklahoma Medical Research Foundation, Oklahoma City, Oklahoma; Department of Medicine, State University of New York (SUNY) Downstate Medical Center, Brooklyn, New York; Department of Medicine, Division of Clinical Immunology and Rheumatology, University of Alabama at Birmingham, Birmingham, Alabama; Department of Rheumatology, Johns Hopkins University School of Medicine, Baltimore, Maryland; Feinstein Institute for Medical Research, Manhasset, New York; Division of Rheumatology and Immunology, Department of Medicine, University of North Carolina, Chapel Hill, North Carolina; Autoimmunity Institute, Allegheny Health Network, Pittsburgh, Pennsylvania; Northwestern University and Feinberg School of Medicine, Chicago, Illinois; Division of Rheumatology, Emory University School of Medicine, Atlanta, Georgia; University of California San Diego School of Medicine, La Jolla, California; Division of Rheumatology, Medical University of South Carolina, Charleston, South Carolina; Division of Rheumatology, Columbia University Medical Center, New York, New York, USA; Department of Rheumatology, Center for Rheumatology Research Fossvogur Landspitali University Hospital, Reyjkavik, Iceland; Autoimmune Disease Unit, Department of Internal Medicine, Hospital Universitario Cruces, BioCruces Health Research Institute, University of the Basque Country, Barakaldo; Josep Font Autoimmune Diseases Laboratory, Institut d'Investigacions Biomèdiques August Pi i Sunyer (IDIBAPS), Department of Autoimmune Diseases, Hospital Clínic, Barcelona, Spain; Division of Rheumatology, Department of Internal Medicine, Istanbul Medical Faculty, Istanbul University, Istanbul, Turkey; Copenhagen Lupus and Vasculitis Clinic, Centre for Rheumatology and Spine Diseases, Rigshospitalet, Copenhagen University Hospital, Copenhagen, Denmark; Department of Rheumatology, Kantousspital, Schaffhausen, Switzerland.

This work was supported by grants from the Swedish Rheumatism Association, the County Council of Östergötland, the Swedish Society of Medicine, the King Gustaf V and Queen Victoria's Freemasons foundation, and the King Gustaf V's 80-year anniversary foundation. Dr. Fortin holds a Canada Research Chair on Systemic Autoimmune Rheumatic Diseases. Dr. Bae's work was supported in part by an unrestricted grant (Hanyang University 201600000001387). Dr. Gordon's work was supported by Lupus UK and the NIHR/Wellcome Trust Clinical Research Facility. The Hopkins Lupus Cohort is supported by the US National Institutes of Health (NIH; grant AR43727). The Montreal General Hospital Lupus Clinic is partially supported by the Singer Family Fund for Lupus Research.Dr. Clarke holds The Arthritis Society Chair in Rheumatic Diseases at the University of Calgary. Dr. Bruce is supported by Arthritis Research UK, the NIHR Manchester Biomedical Research Centre and the NIHR/Wellcome Trust Clinical Research Facility at Manchester University National Health Service (NHS) Foundation Trust. The views expressed in this publication are those of the author( $s$ ) and not necessarily those of the NHS, the NIHR, or the Department of Health. Dr. Jacobsen is supported by the Danish Rheumatism Association (A1028). Dr. Dooley's work was supported by NIH grant RR00046.

L. Wirestam, PhD, Rheumatology/Division of Neuro and Inflammation Sciences, Department of Clinical and Experimental Medicine, Linköping University; H. Enocsson, PhD, Rheumatology/Division of Neuro and Inflammation Sciences, Department of Clinical and Experimental Medicine, Linköping University; T. Skogh, MD, PhD,

Rheumatology/Division of Neuro and Inflammation Sciences, Department of Clinical and Experimental Medicine, Linköping University;

L. Padyukov, MD, PhD, Department of Medicine, Unit of Rheumatology, Karolinska Institutet and Karolinska University Hospital; A. Jönsen, MD, PhD, Department of Clinical Sciences Lund, Section of Rheumatology, Lund University; M.B. Urowitz, MD, FRCPC, Professor of Medicine, Centre for Prognosis Studies in the Rheumatic Diseases, Toronto Western Hospital and University of Toronto; D.D. Gladman, MD, FRCPC, Professor of Medicine, Centre for Prognosis Studies in the Rheumatic Diseases, Toronto Western Hospital and University of Toronto; J. Romero-Diaz, MD, MSc, Instituto Nacional de Ciencias Médicas y
Nutrición; S.C. Bae, MD, Department of Rheumatology, Hanyang University Hospital for Rheumatic Diseases; P.R. Fortin, MD, MPH, FRCPC, Professor of Medicine, Division of Rheumatology, CHU de Québec - Université Laval; J. Sanchez-Guerrero, MD, MSc, Centre for Prognosis Studies in the Rheumatic Diseases, Toronto Western Hospital and University of Toronto; A.E. Clarke, MD, MSc, Division of Rheumatology, Cumming School of Medicine-University of Calgary; S. Bernatsky, MD, PhD, FRCPC, Professor of Medicine, Division of Rheumatology, Department of Medicine, McGill University Health Centre; C. Gordon, MD, Rheumatology Research Group, School of Immunity and Infection, College of Medical and Dental Sciences, University of Birmingham; J.G. Hanly, MD, Division of Rheumatology, Department of Medicine and Department of Pathology, Queen Elizabeth II Health Sciences Centre and Dalhousie University; D. Wallace, MD, Cedars-Sinai/David Geffen School of Medicine, University of California Los Angeles; D.A. Isenberg, MD, Centre for Rheumatology Research, University College London; A. Rahman, MD, PhD, Centre for Rheumatology Research, University College London; J. Merrill, MD, Department of Clinical Pharmacology, Oklahoma Medical Research Foundation; E. Ginzler, MD, PhD, Department of Medicine, SUNY Downstate Medical Center; G.S. Alarcón, MD, MPH, Department of Medicine, Division of Clinical Immunology and Rheumatology, University of Alabama at Birmingham; W.W. Chatham, MD, Department of Medicine, Division of Clinical Immunology and Rheumatology, University of Alabama at Birmingham; M. Petri, MD, Department of Rheumatology, Johns Hopkins University School of Medicine; M. Khamashta, MD, Lupus Research Unit, The Rayne Institute, St. Thomas' Hospital, King's College London School of Medicine; C. Aranow, MD, Feinstein Institute for Medical Research; M. Mackay, MD, Feinstein Institute for Medical Research; M.A. Dooley, MD, MPH, Division of Rheumatology and Immunology, Department of Medicine, University of North Carolina; S. Manzi, MD, MPH, Autoimmunity Institute, Allegheny Health Network; R. Ramsey-Goldman, MD, DrPH, Northwestern University and Feinberg School of Medicine; O. Nived, MD, PhD, Department of Clinical Sciences Lund, Section of Rheumatology, Lund University; K. Steinsson, MD, Department of Rheumatology, Center for Rheumatology Research Fossvogur Landspitali University Hospital; A. Zoma, MD, Lanarkshire Centre for Rheumatology, Hairmyres Hospital; G. Ruiz-Irastorza, MD, Autoimmune Disease Unit, Department of Internal Medicine, Hospital Universitario Cruces, BioCruces Health Research Institute, University of the Basque Country; S. Lim, MD, MPH, Division of Rheumatology, Emory University School of Medicine; K. Kalunian, MD, University of California San Diego School of Medicine; M. Inanc, MD, Division of Rheumatology, Department of Internal Medicine, Istanbul Medical Faculty, Istanbul University; R. van Vollenhoven, MD, ClinTRID, Karolinska University; M. Ramos-Casals, MD, Josep Font Autoimmune Diseases Laboratory, IDIBAPS, Department of Autoimmune Diseases, Hospital Clínic; D.L. Kamen, MD, Division of Rheumatology, Medical University of South Carolina; S. Jacobsen, MD, DMSc, Copenhagen Lupus and Vasculitis Clinic, Centre for Rheumatology and Spine Diseases, Rigshospitalet, Copenhagen University Hospital; C. Peschken, MD, FRCPC, Associate Professor of Medicine, Department of Medicine and Community Health Sciences, University of Manitoba; A. Askanase, MD, MPH, Division of Rheumatology, Columbia University Medical Center; T. Stoll, MD, Department of Rheumatology, Kantousspital; I.N. Bruce, MD, Arthritis Research UK Centre for Epidemiology, Centre for Musculoskeletal Research, Faculty of Biology, Medicine and Health, The University of Manchester and NIHR Manchester Biomedical Research Centre, Manchester University Foundation Trust; J. Wetterö, PhD, Rheumatology/Division of Neuro and Inflammation Sciences, Department of Clinical and Experimental Medicine, Linköping University; C. Sjöwall, $M D, P h D$, Rheumatology/Division of Neuro and Inflammation Sciences, Department of Clinical and Experimental Medicine, Linköping University.

Address correspondence to Dr. L. Wirestam, AIR/Rheumatology, Department of Clinical and Experimental Medicine, Campus US, Linköping University, SE-581 85 Linköping, Sweden. E-mail: lina.wirestam@gmail.com

Full Release Article. For details see Reprints and Permissions at jrheum.org

Accepted for publication October 4, 2018. 
Systemic lupus erythematosus (SLE) is a multisystemic inflammatory rheumatic disease that often shows periods of flares followed by remission. Distinguishing ongoing inflammation attributed to SLE from established organ damage caused by the disease, medication, or comorbidities remains a challenge for the clinician. The spectrum of phenotypes complicates the search for biomarkers that adequately reflect active disease and/or increasing organ damage.

Osteopontin (OPN), an extracellular matrix protein with multiple functions, has been reported to be involved in inflammation ${ }^{1}$. Local production and elevated circulating levels of OPN have been observed in several autoimmune diseases, such as multiple sclerosis ${ }^{2}$, rheumatoid arthritis ${ }^{3}$, and $\mathrm{SLE}^{4,5}$. Overexpression of OPN in lupus-prone mice induces B-cell activation and subsequent production of anti-dsDNA antibodies ${ }^{6,7}$, a hallmark of SLE. Intracellular OPN has been implicated in numerous cellular processes and its expression is required for Toll-like receptor 9 (TLR-9)dependent production of interferon $\alpha(\text { IFN }-\alpha)^{8}$, a central cytokine in the SLE pathogenesis 9 .

Elevated OPN levels have been found to distinguish SLE from healthy individuals $s^{4,5,10}$. Further, associations between OPN and SLE disease activity ${ }^{11}$ as well as with organ damage accrual ${ }^{12}$ have been reported. In addition, elevated OPN levels have been suggested to precede the development of organ damage in a study including predominantly pediatric SLE cases ${ }^{13}$. We have previously investigated serum OPN in a cross-sectional Swedish SLE cohort in which OPN appeared to reflect current global organ damage ${ }^{4}$. OPN was also found to associate with lupus nephritis, antiphospholipid syndrome (APS), and individual clinical and laboratory criteria of APS. In addition, OPN levels showed significant correlations with SLE disease activity, particularly in newly diagnosed cases.

The aims of our study were to determine whether OPN (1) predicts future organ damage, (2) reflects current and/or persistent disease activity, and (3) associates with certain disease phenotypes, using a longitudinal international inception cohort of recent-onset SLE.

\section{MATERIALS AND METHODS}

Systemic Lupus International Collaborating Clinics (SLICC) Inception Cohort. The SLICC Inception Cohort was recruited from 31 centers in 11 countries in North America, Europe, and Asia from 2000 to 2011, as previously described ${ }^{14,15}$. Briefly, all clinical data were submitted to the coordinating center at the University of Toronto and patients were reviewed annually. Laboratory tests necessary to evaluate disease activity, including complement proteins and autoantibodies, and variables related to organ damage were performed at the recruiting centers, Exceptions for this were OPN and estimated glomerular filtration rate (eGFR) based on serum creatinine.

Patients and controls. SLE cases were enrolled within 15 months (mean 6 mos, range 0-15) of SLE diagnosis, which was based on the fulfillment of at least 4 of the American College of Rheumatology (ACR) 1997 criteria $^{16}$. We selected patients from the inception cohort who had baseline serum available and for which there were 5 years of annual followup data completed. In addition, absence of organ damage at baseline was a requirement. At each visit, these measures were assessed: Systemic Lupus Erythematosus Disease Activity Index 2000 (SLEDAI-2K) ${ }^{17}$, clinical SLEDAI (scores for complement consumption and increased DNA binding subtracted from SLEDAI-2K), serological activity (scores for complement consumption and increased DNA binding only), and SLICC/ACR damage index (SDI) ${ }^{18}$. Patients with "persistent disease activity" were defined as having SLEDAI-2K scores of $\geq 5$ at $\geq 3$ separate occasions during the 5 -year followup. At baseline, peripheral venous blood was drawn from each individual. Sera were prepared and stored at $-70^{\circ} \mathrm{C}$ until analyzed.

Sera from population-based controls matched 1:1 according to sex and age included in the Swedish Epidemiological Investigation of Rheumatoid Arthritis (EIRA) cohort served as controls for the OPN analyses ${ }^{19}$.

This study was approved by the SLICC data coordinating center's institutional research ethics board at the University Health Network (file no. 00-0279). Each of the 33 participating centers' institutional research ethics boards approved the SLICC inception cohort study.

OPN immunoassay. A serum- and plasma-validated ELISA kit (Quantikine, R\&D Systems) was used to analyze OPN levels in SLE and control sera. All OPN assays were performed in Linköping (Sweden), and the analyses were in accordance with the manufacturers' instructions. Briefly, serum (diluted $1: 25)$ was added to microwells pre-coated with monoclonal antibodies directed against human OPN. After incubation and washing, a horseradish-peroxide conjugated polyclonal anti-OPN antibody was added and the plate incubated, followed by the washing and addition of tetramethylbenzidine substrate. The enzymatic reaction was stopped by adding $2 \mathrm{~N}$ sulfuric acid and read at $450 \mathrm{~nm}$ (plate reader: Sunrise, Tecan; software: Magellan version 7.1, Tecan).

Creatinine and $e G F R$. Serum creatinine was determined using an enzymatic colorimetric method at the clinical chemistry laboratory (Linköping University Hospital, Sweden). The 4-variable Modification of Diet in Renal Disease Study equation was used to calculate eGFR ${ }^{20}$.

Statistics. Sample size calculation (for comparing 2 groups) revealed that sera from 208 individuals were needed to detect a significant difference in OPN levels between SLE patients with versus without any organ damage at followup. This calculation was based on the following: (1) a power of $80 \%$; (2) an SD of $36.8 \mathrm{ng} / \mathrm{ml}$, which was the OPN level (SD) in patients with permanent organ damage using data from our pilot study ${ }^{4}$; and (3) the approximation that at least $25 \%$ of the patients with SLE would develop any kind of organ damage during the 5-year followup.

Independent samples $t$ tests were used to evaluate differences in OPN levels between SLE patients and controls, and between patients meeting and not meeting specific ACR criteria.

Pearson correlation analyses were performed between OPN and disease activity measures (erythrocyte sedimentation rate, SLEDAI-2K, clinical SLEDAI, and serological activity) as well as between OPN and the total number of fulfilled ACR criteria. Significant associations were further analyzed in a univariate general linear model (GLM) with adjustment for age, sex, race/ethnicity, and daily glucocorticoid (GC) dose at baseline. In addition, the association between OPN and nephritis was adjusted for eGFR

ANOVA was used to evaluate differences in OPN levels between patients with "no damage," "moderate damage," and "extensive damage."

Binary logistic regression was used to predict damage accrual (global SDI, as well as organ domains of SDI) with adjustments for baseline data on age, sex, race/ethnicity, SLEDAI-2K, and GC therapy. Binary logistic regression was used to predict persistent disease activity with adjustments for baseline data on age, sex, race/ethnicity, and GC therapy.

Statistical significance was set at $\mathrm{p}<0.05$, along with $95 \%$ CI. Statistical analyses were performed with SPSS Statistics 22 (IBM) or GraphPad Prism, version 5.04 (GraphPad Software).

\section{RESULTS}

The study included 344 SLE cases ( 315 women and 29 men; mean age $34.0 \mathrm{yrs}$, range $12-73$ ). The majority of patients

Personal non-commercial use only. The Journal of Rheumatology Copyright $\odot$ (2019. All rights reserved 
( $n=200,58 \%)$ were of white ethnicity. Of the 344 controls (315 women and 29 men; mean age 34.4 yrs, range 15-73 yrs), 327 (95\%) were of white race/ethnicity. Detailed characteristics of the study populations are found in Table 1.

Baseline OPN levels are increased in SLE. Circulating levels of OPN were markedly higher in patients with SLE (mean $45.4 \mathrm{ng} / \mathrm{ml}, 95 \%$ CI $41.4-49.4$ ) than in the controls (mean $11.8 \mathrm{ng} / \mathrm{ml}, 95 \%$ CI 10.4-13.3, $\mathrm{p}<0.0001$; Figure 1A). OPN levels correlated inversely with age, both among the patients $(\mathrm{r}=-0.17, \mathrm{p}=0.002)$ and the controls $(\mathrm{r}=-0.27, \mathrm{p}<0.0001)$. No differences were observed between men and women among the controls regarding OPN levels. However, among patients with SLE, men displayed higher OPN levels (mean $79.5 \mathrm{ng} / \mathrm{ml}, 95 \%$ CI 47.0-111.9) compared to women (mean $42.3 \mathrm{ng} / \mathrm{ml}, 95 \%$ CI $39.1-45.4, \mathrm{p}<0.0001)$. There were no significant differences in baseline disease activity (SLEDAI-2K) between men (mean 4.3, 95\% CI 2.77-5.9)

Table 1. Baseline characteristics of the 344 SLE patients and 344 population-based controls.

\begin{tabular}{|c|c|c|}
\hline Characteristics & Patients with SLE & Controls \\
\hline \multicolumn{3}{|l|}{ Background variables } \\
\hline Age, yrs & $34.0(12-73)$ & $34.4(15-73)$ \\
\hline Weight, kg & $67.5(32.6-133.0)$ & NA \\
\hline Height, $\mathrm{cm}$ & $164.6(145.0-194.5)$ & NA \\
\hline Female sex & $315(91.6)$ & $315(91.6)$ \\
\hline \multicolumn{3}{|l|}{ Ethnicities } \\
\hline White & $200(58.1)$ & $327(95.1)$ \\
\hline African descendants & $52(15.1)$ & $4(1.1)$ \\
\hline Asian & $64(18.6)$ & $3(0.9)$ \\
\hline Other & $28(8.1)$ & $10(2.9)$ \\
\hline \multicolumn{3}{|l|}{ Disease variables } \\
\hline SLEDAI-2K score & $5.0(0-30)$ & NA \\
\hline C-reactive protein, $\mathrm{mg} / \mathrm{l}^{*}$ & $4.6(0-114)$ & \\
\hline Erythrocyte sedimentation rate, $\mathrm{mm} / \mathrm{h}^{\#}$ & $23.9(1-99)$ & \\
\hline Glucocorticoid dose at baseline, $\mathrm{mg} / \mathrm{da}$ & ay $14(0-90)$ & \\
\hline Creatinine, mg/dl & $0.7(0.1-8.1)$ & \\
\hline $\mathrm{eGFR}, \mathrm{ml} / \mathrm{min} / 1.73 \mathrm{~m}^{2}$ & $119.9(6.3-473.2)$ & \\
\hline Low complement & $130(37.8)$ & \\
\hline Increased DNA binding & $150(43.6)$ & \\
\hline 1997 ACR criteria (at enrollment) & & NA \\
\hline 1. Malar rash & $111(32.3)$ & \\
\hline 2. Discoid rash & $39(11.3)$ & \\
\hline 3. Photosensitivity & $131(38.1)$ & \\
\hline 4. Oral ulcers & $125(36.3)$ & \\
\hline 5. Arthritis & $249(72.4)$ & \\
\hline 6. Serositis & $92(26.7)$ & \\
\hline 7. Renal disorder & $75(21.8)$ & \\
\hline 8. Neurologic disorder & $12(3.5)$ & \\
\hline 9. Hematologic disorder & $214(62.2)$ & \\
\hline 10. Immunologic disorder & $265(77.0)$ & \\
\hline 11. Antinuclear antibody & $336(97.7)$ & \\
\hline
\end{tabular}

Data are presented as mean (range) or $\mathrm{n}(\%) .{ }^{*}$ Performed locally at each SLICC centre $(n=312)$. " Performed locally at each SLICC centre $(n=167)$. ACR: American College of Rheumatology; SLE: systemic lupus erythematosus; SLEDAI-2K: SLE Disease Activity Index 2000; eGFR: estimated glomerular filtration rate; SLICC: Systemic Lupus International Collaborating Clinics; NA: not applicable. and women (mean 5.0, 95\% CI 4.5-5.6, $\mathrm{p}=0.45$ ). However, clear differences in OPN levels were identified between patients of white race/ethnicity (mean $38.2 \mathrm{ng} / \mathrm{ml}, 95 \%$ CI $34.7-41.7$ ) compared to non-whites (mean $55.4 \mathrm{ng} / \mathrm{ml}, 95 \%$ CI 47.4-63.5, $\mathrm{p}<0.0001)$. Such a difference was not found among the controls (whites: mean $11.8 \mathrm{ng} / \mathrm{ml}, 95 \% \mathrm{CI}$ 10.3-13.3; non-whites: mean $12.3 \mathrm{ng} / \mathrm{ml}, 95 \%$ CI $5.5-19.1$; $\mathrm{p}=0.87)$. Patients of non-white race/ethnicity had higher disease activity (mean 6.1, 95\% CI 5.2-7.0) compared to whites (mean $4.1,95 \%$ CI 3.5-4.7, $\mathrm{p}=0.0002$ ).

OPN failed to predict damage accrual in adjusted analyses. At the 3-year followup visit, 63 patients (18\%) with SLE had developed any damage (i.e., SDI $\geq 1$ ), and 98 (29\%) showed damage after 5 years. Because only $18 \%$ had an SDI score of $\geq 1$ three years post-inclusion, we focused mainly on the 5 -year data. A weak correlation was found between baseline OPN and damage accrual after 5 years $(r=0.15, p=0.006)$. However, in a binary logistic regression analysis with adjustments, OPN levels failed to predict future global damage when defined as SDI $\geq 1$ with a receiver-operating characteristic area under curve (AUC) of $0.67(\mathrm{p}=0.061$; Table 2). Examining each domain of SDI separately rendered no statistically significant association with OPN levels. However, age and SLEDAI-2K at baseline significantly predicted organ damage development at 5 years (Table 2).

We did not identify any significant differences in baseline OPN levels when separating patients' SDI after 5 years into "no damage" (i.e., SDI = 0, $\mathrm{n}=246$, mean $41.9 \mathrm{ng} / \mathrm{ml}, 95 \%$ CI 38.6-45.3), "moderate damage" (SDI 1-2, $\mathrm{n}=84$, mean $52.5,95 \%$ CI 40.0-65.1), and "extensive damage" (SDI $\geq 3$, $\mathrm{n}=14$, mean $63.4 \mathrm{ng} / \mathrm{ml}, 95 \%$ CI 38.6-88.2).

OPN reflects disease activity and renal involvement. Baseline OPN correlated with SLEDAI-2K $(\mathrm{r}=0.27, \mathrm{p}<0.0001)$, clinical SLEDAI $(\mathrm{r}=0.22, \mathrm{p}<0.0001)$, and serological activity $(\mathrm{r}=0.24, \mathrm{p}<0.0001)$ at enrollment into the cohort. Using a binary variable for anti-dsDNA (positive/negative) showed that patients positive for anti-dsDNA had significantly higher OPN levels (mean $55.7 \mathrm{ng} / \mathrm{ml}, 95 \%$ CI $48.2-63.2, \mathrm{n}=150)$ compared to those that were negative (mean $37.4 \mathrm{ng} / \mathrm{ml}, 95 \%$ CI 33.6-41.2, $\mathrm{n}=194$ ), $\mathrm{p}<0.0001$. Patients with low complement (C3 and/or C4) had higher levels of OPN (mean $54.7 \mathrm{ng} / \mathrm{ml}, 95 \%$ CI 46.6-62.8, $\mathrm{n}=130$ ) compared to those with normal complement (mean 39.7 $\mathrm{ng} / \mathrm{ml}, 95 \%$ CI 35.7-43.8, $\mathrm{n}=214 ; \mathrm{p}=0.0003$ ). Patients with a SLEDAI-2K score of $\geq 5$ had higher levels of OPN (mean $56.6 \mathrm{ng} / \mathrm{ml}$, 95\% CI 48.3-64.9) than patients with SLEDAI-2K < 5 (mean $38.5 \mathrm{ng} / \mathrm{ml}, 95 \%$ CI $34.7-42.2 ; \mathrm{p}<$ 0.0001 ; Figure 1B). The erythrocyte sedimentation rate correlated with OPN $(r=0.38, p<0.0001)$. The above-mentioned associations remained significant after adjustments for age, sex, race/ethnicity, and GC therapy in a univariate GLM analysis.

We further evaluated associations with different disease phenotypes (i.e., fulfilled ACR criteria). Only the renal

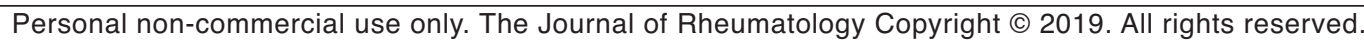


A

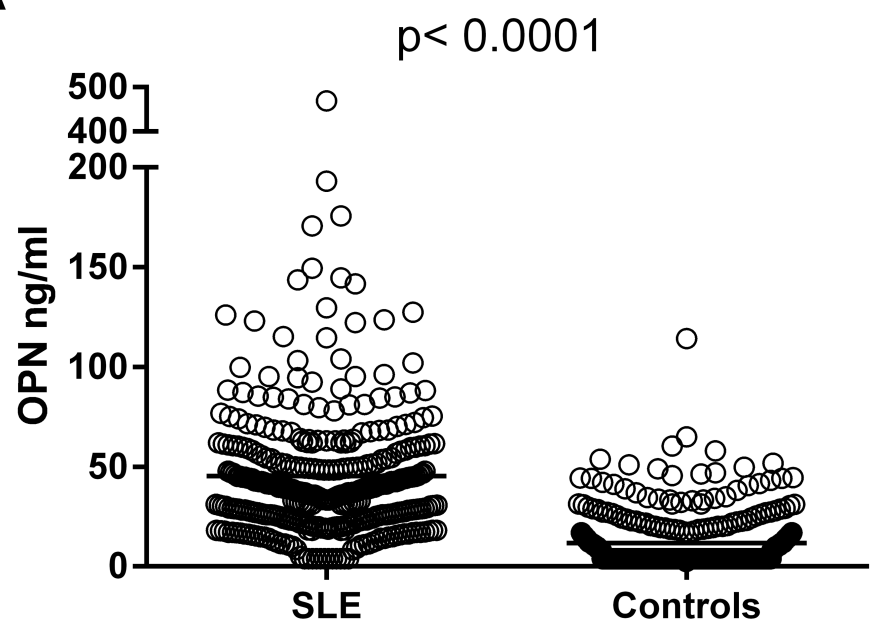

B

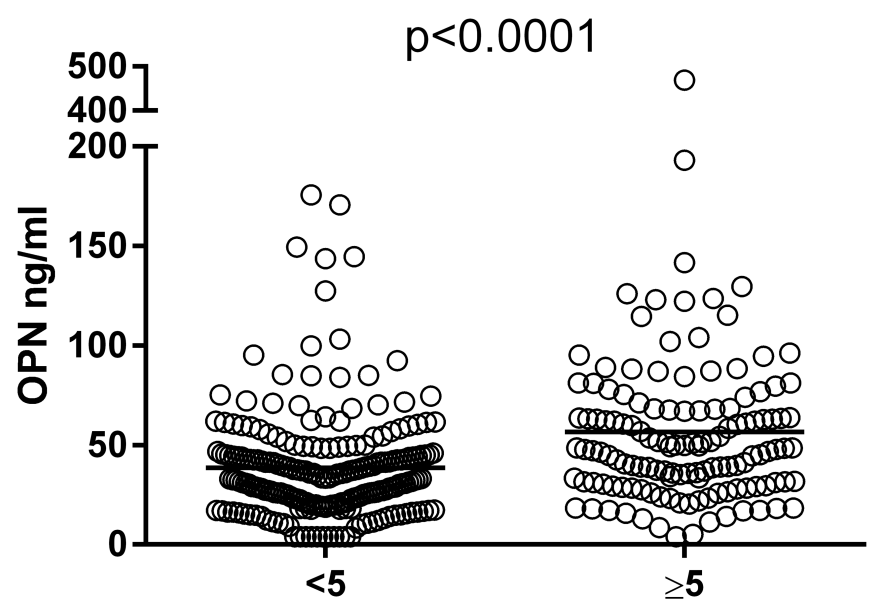

SLEDAI-2K at baseline

Figure 1. Serum osteopontin (OPN) levels. A. Baseline levels of OPN were significantly higher among patients with SLE (mean $45.4 \mathrm{ng} / \mathrm{ml}, \mathrm{n}=344$ ) compared to controls (mean $11.8 \mathrm{ng} / \mathrm{ml}, \mathrm{n}=344$ ). B. Patients with raised disease activity (SLEDAI-2K $\geq 5$ ) had higher baseline levels of OPN (mean $56.6 \mathrm{ng} / \mathrm{ml}, \mathrm{n}=131$ ) than patients with low/no disease activity (SLEDAI-2K $<5$; mean $38.5 \mathrm{ng} / \mathrm{ml}, \mathrm{n}=213$ ). SLE: systemic lupus erythematosus; SLEDAI-2K: SLE Disease Activity Index 2000.

Table 2. Binary logistic regression for the outcome of organ damage (SLICC/ACR Damage Index $\geq 1$; yes/no) at 5 years.

\begin{tabular}{lcc}
\hline Variables & OR $(95 \% \mathrm{CI})$ & $\mathrm{p}$ \\
\hline OPN at baseline & $1.01(1.00-1.02)$ & 0.061 \\
Age at baseline & $1.03(1.01-1.05)$ & 0.006 \\
Female sex & $0.50(0.22-1.18)$ & 0.115 \\
White ethnicity & $0.87(0.72-1.04)$ & 0.127 \\
Daily glucocorticoid dose at baseline & $0.99(0.97-1.01)$ & 0.241 \\
SLEDAI-2K at baseline & $1.07(1.02-1.13)$ & 0.013
\end{tabular}

SLICC/ACR: Systemic Lupus International Collaborating Clinics/American College of Rheumatology; OPN: osteopontin; SLEDAI-2K: Systemic Lupus Erythematosus Disease Activity Index 2000. disorder criterion (ACR-7) reached statistical significance with higher levels of OPN (mean $63.7 \mathrm{ng} / \mathrm{ml}, 95 \% \mathrm{CI}$ 49.9-77.5, $\mathrm{n}=75)$ compared to those without renal involvement (mean $40.3 \mathrm{ng} / \mathrm{ml}, 95 \%$ CI 37.1-43.5, $\mathrm{n}=269$; $\mathrm{p}<0.0001$; Figure 2A). The association with nephritis remained significant after adjustments for age, sex, race/ethnicity, GC therapy, and eGFR in a univariate GLM analysis. Eighty-three patients had an impaired eGFR $(\leq 90$ $\mathrm{ml} / \mathrm{min} / 1.73 \mathrm{~m}^{2}$ ), but only 12 patients had an eGFR $<60$. Higher levels of OPN were found in patients with an impaired eGFR (mean $54.6 \mathrm{ng} / \mathrm{ml}, 95 \%$ CI 42.3-66.9, $\mathrm{n}=83$ ) compared to those with eGFR $>90$ (mean $42.5,95 \%$ 
A

$\mathrm{p}<0.0001$

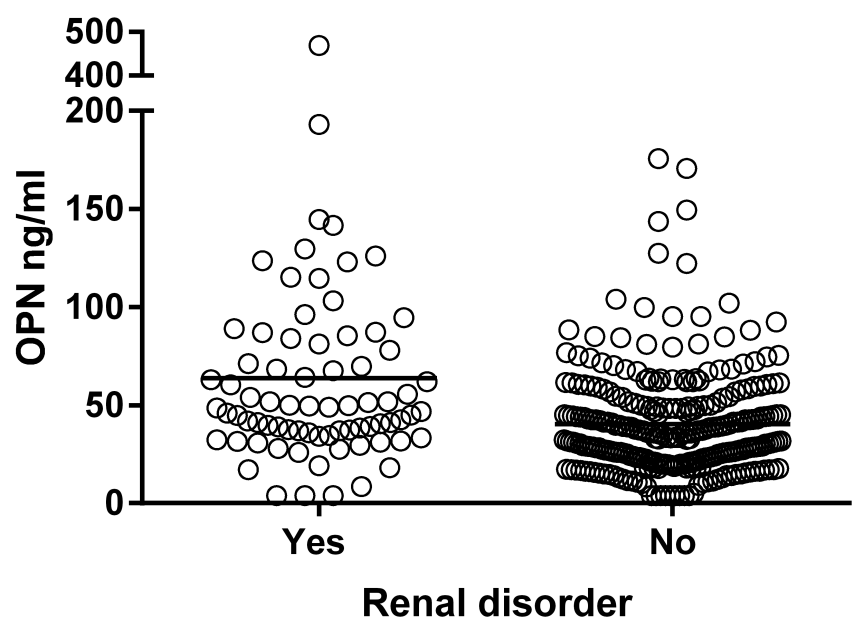

B

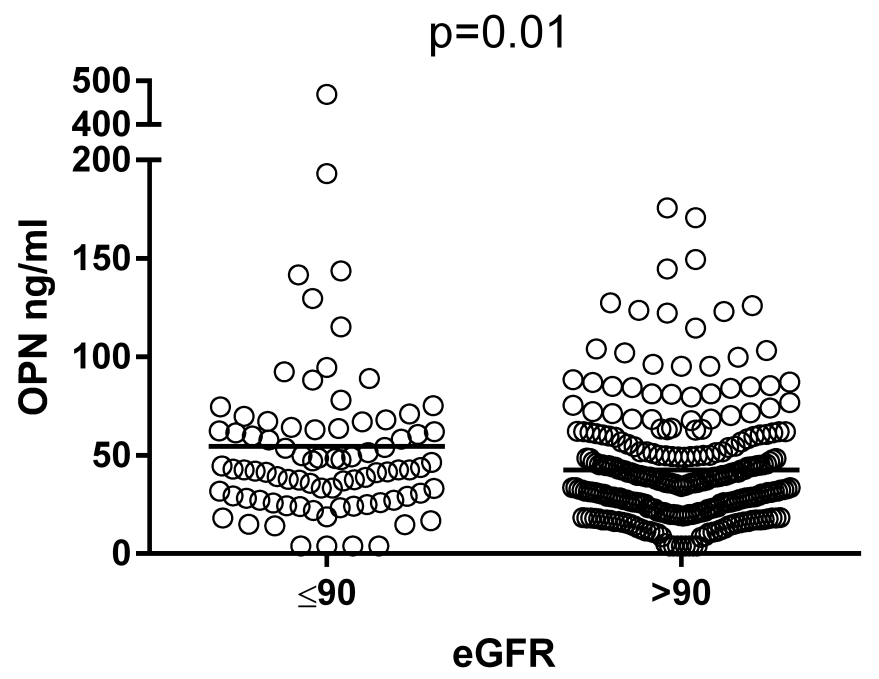

Figure 2. Serum osteopontin (OPN) levels in SLE cases with or without renal involvement. A. Patients meeting the renal disorder criterion (ACR-7) had significantly higher baseline levels of OPN (mean $63.7 \mathrm{ng} / \mathrm{ml}, \mathrm{n}=75$ ) compared to those without renal involvement (mean $40.3 \mathrm{ng} / \mathrm{ml}, \mathrm{n}=269$ ). B. Higher OPN levels were found in patients with impaired eGFR (mean $54.6, \mathrm{n}=83$ ) compared to those with normal eGFR (mean 42.5, $\mathrm{n}=261$ ). SLE: systemic lupus erythematosus; ACR: American College of Rheumatology; eGFR: estimated glomerular filtration rate.

CI $38.9-46.0, \mathrm{n}=261 ; \mathrm{p}=0.01$; Figure 2B). Of the 75 patients meeting the renal ACR criterion, patients with an impaired eGFR had higher OPN levels (mean $96.8 \pm 24.5$ $\mathrm{ng} / \mathrm{ml}, \mathrm{n}=18$ ) compared to those with normal eGFR (mean $53.3 \pm 4.2, \mathrm{n}=57 ; \mathrm{p}=0.006$ ). A weak correlation between OPN levels and the total number of fulfilled ACR criteria $(\mathrm{r}=0.17, \mathrm{p}=0.001)$ was identified.

$O P N$ predicts persistent disease activity. To further examine the association between OPN and disease activity, we separated patients based on persistent disease activity (defined as SLEDAI-2K scores of $\geq 5$ at $\geq 3$ separate occasions during the 5-yr followup). Higher levels of OPN were found among the 51 patients $(15 \%)$ with persistent disease activity (mean $62.0 \mathrm{ng} / \mathrm{ml}$, 95\% CI 43.8-80.5) compared to those without (mean $42.5 \mathrm{ng} / \mathrm{ml}, 95 \%$ CI $39.08-45.9 ; \mathrm{p}=0.0006$; Figure $3 \mathrm{~A}$ ). To evaluate the possible effect of organ damage on OPN levels in cases with persistent disease activity $(\mathrm{n}=51)$, those patients who had developed any damage (i.e., SDI $\geq 1)$ after 5 years $(n=18)$ were compared to those without any damage $(n=33)$. No statisti-

Personal non-commercial use only. The Journal of Rheumatology Copyright @ 2019 . All rights reserved. 


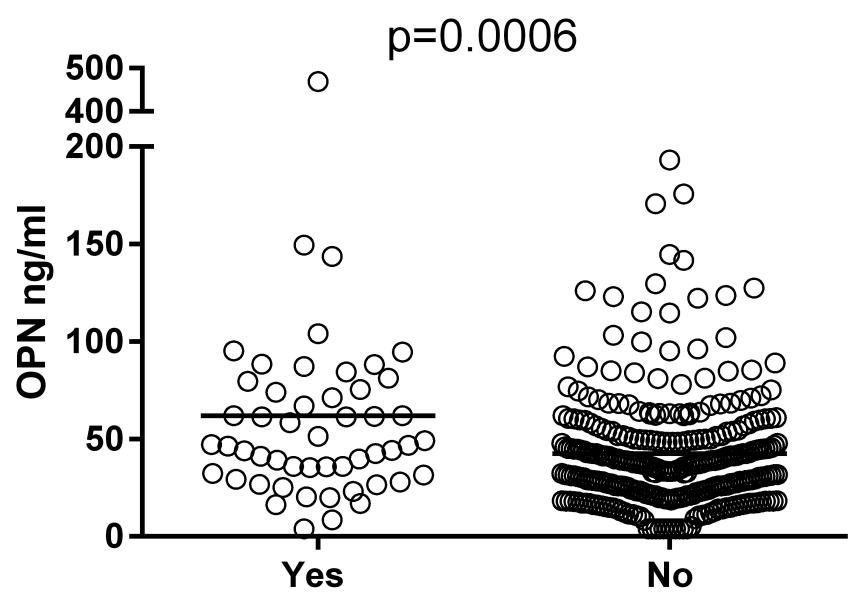

Cases with SLEDAI $\geq 5$ at $\geq 3$ occasions

B

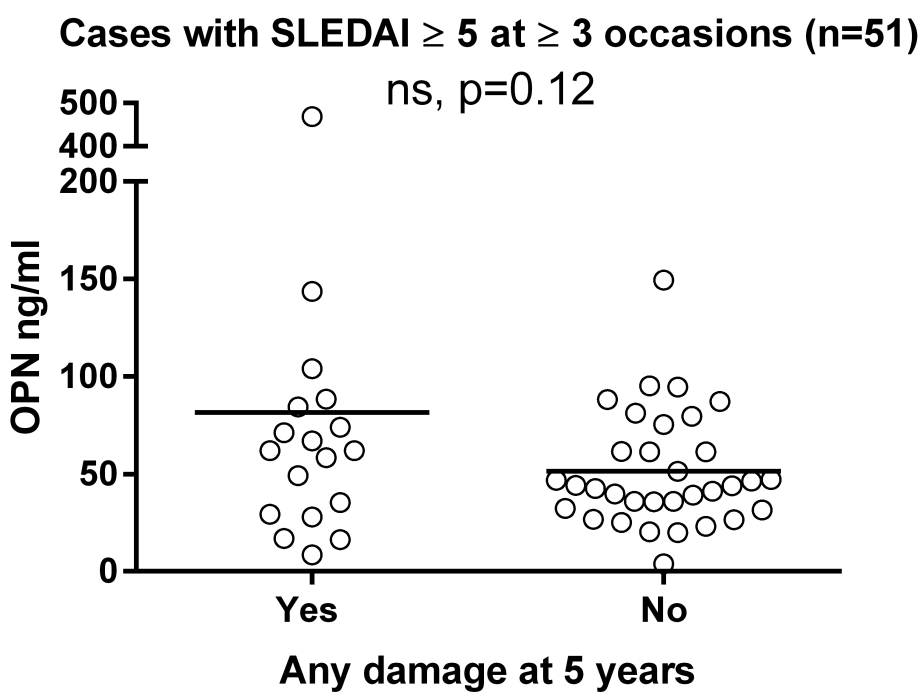

\begin{abstract}
Figure 3. Baseline osteopontin (OPN) levels in patients with persistent disease activity. A. Higher levels of OPN were found in the 51 patients with persistent disease activity (mean $62.0 \mathrm{ng} / \mathrm{ml}$ ) compared to those without (mean $42.5 \mathrm{ng} / \mathrm{ml}, \mathrm{n}=293$ ). B. To investigate the possible effect of damage on OPN levels in cases with persistent disease activity, we compared patients who had developed any damage (i.e., SDI $\geq 1$ ) after 5 years to those without any damage. No significant difference in OPN levels was observed between patients with any damage (mean $81.5 \mathrm{ng} / \mathrm{ml}, \mathrm{n}=18$ ) compared with those without (mean $51.4 \mathrm{ng} / \mathrm{ml}$, $\mathrm{n}=33$ ). SDI: Systemic Lupus International Collaborating Clinics/American College of Rheumatology Damage Index; SLEDAI: Systemic Lupus Erythematosus Disease Activity Index.
\end{abstract}

cally significant difference in OPN levels was observed (Figure 3B).

Using a binary logistic regression model with adjustments, OPN levels were associated with persistent disease activity $(\mathrm{p}=0.011$, AUC $=0.66$; Table 3). Further adjustment for damage (SDI) at 5 years did not change this association $(\mathrm{p}=0.012, \mathrm{AUC}=0.66)$.

\section{DISCUSSION}

In SLE, OPN has been proposed as a useful biomarker of disease activity ${ }^{4,11}$, as well as of organ damage ${ }^{4,12,13}$. Most previous studies had a cross-sectional design, but in our present study we aimed to dissect whether baseline OPN levels could be predictive of future organ damage in a longitudinal cohort. Our results confirm some of the previous 
Table 3. Binary logistic regression for the outcome of persistent disease activity (yes/no) at 5 years.

\begin{tabular}{lcc}
\hline Variables & OR $(95 \% \mathrm{CI})$ & $\mathrm{p}$ \\
\hline OPN at baseline & $1.01(1.00-1.02)$ & 0.011 \\
Age at baseline & $0.97(0.95-1.00)$ & 0.063 \\
Female sex & $1.96(0.51-7.43)$ & 0.325 \\
White ethnicity & $1.02(0.82-1.28)$ & 0.854 \\
Daily glucocorticoid dose at baseline & $1.00(0.98-1.02)$ & 0.821 \\
\hline
\end{tabular}

OPN: osteopontin.

reports indicating that OPN is associated with disease activity and lupus nephritis, rather than being a marker of future damage progression.

In line with previous findings by our group and others $^{4,5,10}$, OPN levels were elevated in patients with SLE compared with population-based healthy controls. According to Rullo, et al, increased circulating OPN levels have been reported to precede increased "cumulative" disease activity and organ damage in patients with SLE, especially in pediatric SLE ${ }^{13}$. In a cross-sectional pilot study, we evaluated OPN in a cohort of Swedish patients with SLE and found that circulating OPN levels were associated with global organ damage ${ }^{4}$. In our present study, OPN levels at entry into the SLICC cohort were not significantly associated with damage accrual after 5 years, using SDI $\geq 1$ as cutoff. However, a larger group of patients with more extensive damage accrual observed during a longer time period is required to further resolve this issue.

Because OPN showed an inverse correlation with age, and because differences were observed between men and women as well as between whites and non-whites, these factors were adjusted for in the statistical analyses. Rullo, et $a l^{13}$ reported that high circulating OPN levels preceded increased "cumulative" SLE disease activity and organ damage over 12 months. In contrast to their study, the SLICC Inception Cohort consists mainly of adult SLE cases, and there may also be differences between the studies regarding race or ethnicities that could have affected the divergent conclusions of OPN levels as a potential biomarker of future organ damage.

In line with earlier reports, we observed an association between OPN and disease activity, using the SLEDAI- $2 \mathrm{~K}^{4,5}$. In our previous pilot study, we noted a robust correlation between SLEDAI-2K and OPN $(r=0.67, p=0.028)$ when we restricted the analysis to patients with recent-onset disease ${ }^{4}$. In our present study, patients with active disease (i.e., SLEDAI-2K $\geq 5$ ) had higher OPN levels compared to those with no/low disease activity (i.e., SLEDAI-2K < 5), and higher OPN levels were also found in patients with persistent disease activity.

We further investigated associations of baseline OPN with different clinical manifestations. Patients meeting the lupus nephritis criterion displayed higher levels of OPN, which corroborates the finding in our pilot study ${ }^{4}$. Patients with impaired renal function had higher OPN levels, but we did not find an association between OPN and the renal domain of SDI. However, such an association has previously been reported $^{4,11,12}$, and lupus-prone mice with nephritis have been shown to express OPN associated with macrophage infiltration $^{21}$. Further, anti-OPN therapy in nephritic rats reduces albuminuria and invasion of macrophages ${ }^{22}$, and OPN knockout mice have less recruitment of macrophages as well as reduced renal fibrosis 23 .

The reason for elevated OPN in SLE remains unclear, but it could be of relevance to the SLE pathogenesis that the intracellular expression of OPN in plasmacytoid dendritic cells (pDC) is required for TLR-9-dependent production of IFN- $\alpha^{8}$. In addition, mutations in tartrate-resistant acid phosphatase (TRAP) cause spondyloenchondrodysplasia, an unusual recessive disease associated with short stature, brain calcifications, and SLE-like autoimmunity ${ }^{24}$. OPN is a substrate for TRAP, and TRAP has been shown to co-localize and physically interact with OPN in pDC and macrophages ${ }^{25}$. Lack of TRAP leads to hyperphosphorylation of OPN and enhanced TLR-9 signaling in pDC with subsequent IFN- $\alpha$ production, which can cause the SLE-like autoimmunity seen in patients with spondyloenchondrodysplasia. Thus, future studies focusing on potential associations between IFN- $\alpha$ and OPN in SLE are highly warranted.

Our study has several strengths, especially the extremely well-characterized SLE population and the prospective study design using a large international inception cohort of SLE patients with 5 years of followup data. Some limitations should also be mentioned. Although all cases were incident and enrolled up to 15 months from diagnosis (mean time 6 months), it cannot be excluded that the baseline sample may have been taken at a timepoint when the patient already received immunosuppressive therapy or antimalarials. Even though the control subjects were matched according to sex and age, the great majority (95\%) were white, which did not reflect the race/ethnicity distribution of the SLE cases $(58 \%$ white). Thus, it cannot be excluded that this difference, as well as the potential effect of environmental factors, may have influenced the disparity of OPN levels between patients and controls. The relatively small number of damage events over 5 years probably reflects well-controlled patients but generates uncertainties in predicting damage accrual. Finally, OPN was analyzed at baseline only and we acknowledge that the predictive value of OPN for different outcome measures (such as SLE flares or damage accrual) may vary over time in established disease.

In early SLE, OPN is elevated and appears to be associated with renal involvement and higher disease activity at sampling, as well as over time. We found no distinct association with accumulation of organ damage. Based on this, we suggest that raised OPN at SLE onset identify cases with risk of high and persistent disease activity but may not

Personal non-commercial use only. The Journal of Rheumatology Copyright $\odot$ 2019. All rights reserved. 
necessarily lead to accrual of damage within 5 years of followup.

\section{ACKNOWLEDGMENT}

We thank the EIRA Study personnel for providing us with information and sera from healthy controls, and Nicole Anderson for biobank handling and logistics, Charlotte Dahle for facilitating laboratory analyses in Linköping, Sweden, and Lars Valter for advice on statistical analyses.

\section{REFERENCES}

1. Clemente N, Raineri D, Cappellano G, Boggio E, Favero F, Soluri $\mathrm{MF}$, et al. Osteopontin bridging innate and adaptive immunity in autoimmune diseases. J Immunol Res 2016;2016:7675437.

2. Chabas D, Baranzini SE, Mitchell D, Bernard CC, Rittling SR, Denhardt DT, et al. The influence of the proinflammatory cytokine, osteopontin, on autoimmune demyelinating disease. Science 2001;294:1731-5.

3. Ohshima S, Yamaguchi N, Nishioka K, Mima T, Ishii T, Umeshita-Sasai M, et al. Enhanced local production of osteopontin in rheumatoid joints. J Rheumatol 2002;29:2061-7.

4. Wirestam L, Frodlund M, Enocsson H, Skogh T, Wettero J, Sjowall C. Osteopontin is associated with disease severity and antiphospholipid syndrome in well characterised Swedish cases of SLE. Lupus Sci Med 2017;4:e00225.

5. Lee YH, Song GG. Correlation between circulating osteopontin level in systemic lupus erythematosus and disease activity and associations between osteopontin polymorphisms and disease susceptibility: a meta-analysis. Lupus 2017;26:132-8.

6. Iizuka J, Katagiri Y, Tada N, Murakami M, Ikeda T, Sato M, et al. Introduction of an osteopontin gene confers the increase in B1 cell population and the production of anti-DNA autoantibodies. Lab Invest 1998;78:1523-33.

7. Sakamoto K, Fukushima Y, Ito K, Matsuda M, Nagata S, Minato N, et al. Osteopontin in spontaneous germinal centers inhibits apoptotic cell engulfment and promotes anti-nuclear antibody production in lupus-prone mice. J Immunol 2016;197:2177-86.

8. Shinohara ML, Lu L, Bu J, Werneck MB, Kobayashi KS, Glimcher $\mathrm{LH}$, et al. Osteopontin expression is essential for interferon-alpha production by plasmacytoid dendritic cells. Nat Immunol 2006;7:498-506.

9. Ronnblom L, Alm GV, Eloranta ML. The type I interferon system in the development of lupus. Semin Immunol 2011;23:113-21.

10. Wu T, Ding H, Han J, Arriens C, Wei C, Han W, et al. Antibody-array-based proteomic screening of serum markers in systemic lupus erythematosus: a discovery study. J Proteome Res 2016;15:2102-14.

11. Wong CK, Lit LC, Tam LS, Li EK, Lam CW. Elevation of plasma osteopontin concentration is correlated with disease activity in patients with systemic lupus erythematosus. Rheumatology 2005;44:602-6.

12. Quaglia M, Chiocchetti A, Cena T, Musetti C, Monti S, Clemente N et al. Osteopontin circulating levels correlate with renal involvement in systemic lupus erythematosus and are lower in ACE inhibitor-treated patients. Clin Rheumatol 2014;33:1263-71.
13. Rullo OJ, Woo JM, Parsa MF, Hoftman AD, Maranian P, Elashoff DA, et al. Plasma levels of osteopontin identify patients at risk for organ damage in systemic lupus erythematosus. Arthritis Res Ther 2013;15:R18.

14. Bruce IN, O'Keeffe AG, Farewell V, Hanly JG, Manzi S, Su L, et al Factors associated with damage accrual in patients with systemic lupus erythematosus: Results from the systemic lupus international collaborating clinics (SLICC) Inception Cohort. Ann Rheum Dis 2015;74:1706-13.

15. Parker B, Urowitz MB, Gladman DD, Lunt M, Bae SC, Sanchez-Guerrero J, et al. Clinical associations of the metabolic syndrome in systemic lupus erythematosus: Data from an international inception cohort. Ann Rheum Dis 2013;72:1308-14.

16. Hochberg MC. Updating the American College of Rheumatology revised criteria for the classification of systemic lupus erythematosus. Arthritis Rheum 1997;40:1725.

17. Gladman DD, Ibañez D, Urowitz MB. Systemic lupus Erythematosus Disease Activity Index 2000. J Rheumatol 2002;29:288-91.

18. Gladman D, Ginzler E, Goldsmith C, Fortin P, Liang M, Urowitz M et al. The development and initial validation of the Systemic Lupus International Collaborating Clinics/American College of Rheumatology Damage Index for systemic lupus erythematosus. Arthritis Rheum 1996;39:363-9.

19. Stolt P, Bengtsson C, Nordmark B, Lindblad S, Lundberg I, Klareskog L, et al; EIRA study group. Quantification of the influence of cigarette smoking on rheumatoid arthritis: results from a population based case-control study, using incident cases. Ann Rheum Dis 2003;62:835-41.

20. Levey AS, Coresh J, Greene T, Marsh J, Stevens LA, Kusek JW, et al. Expressing the Modification of Diet in Renal Disease Study equation for estimating glomerular filtration rate with standardized serum creatinine values. Clin Chem 2007;53:766-72.

21. Wuthrich RP, Fan X, Ritthaler T, Sibalic V, Yu DJ, Loffing J, et al. Enhanced osteopontin expression and macrophage infiltration in MRL-Fas(lpr) mice with lupus nephritis. Autoimmunity 1998;28:139-50.

22. Panzer U, Thaiss F, Zahner G, Barth P, Reszka M, Reinking RR, et al. Monocyte chemoattractant protein-1 and osteopontin differentially regulate monocytes recruitment in experimental glomerulonephritis. Kidney Int 2001;59:1762-9.

23. Persy VP, Verhulst A, Ysebaert DK, De Greef KE, De Broe ME. Reduced postischemic macrophage infiltration and interstitial fibrosis in osteopontin knockout mice. Kidney Int 2003;63:543-53.

24. Briggs TA, Rice GI, Daly S, Urquhart J, Gornall H, Bader-Meunier $\mathrm{B}$, et al. Tartrate-resistant acid phosphatase deficiency causes a bone dysplasia with autoimmunity and a type I interferon expression signature. Nat Genet 2011;43:127-31.

25. An J, Briggs TA, Dumax-Vorzet A, Alarcon-Riquelme ME, Belot A, Beresford M, et al. Tartrate-resistant acid phosphatase deficiency in the predisposition to systemic lupus erythematosus. Arthritis Rheumatol 2017;69:131-42. 